\title{
An AIDS patient with urine retention
}

\author{
Lijun $\mathrm{Xu}^{1,2^{*}}$ (D), Ran Tao ${ }^{1,2}$, Qiyu Zhao ${ }^{3}$, Jinlin Cheng ${ }^{1,2}$ and Biao Zhu ${ }^{1,2^{*}}$
}

\begin{abstract}
Background: Cryptococcal prostatitis is a rare clinical disease and has never been reported in China.

Case presentation: We report on a male HIV-infected patient with pulmonary and prostate cryptococcosis that was misdiagnosed (as tuberculosis) and delayed diagnosed. Although the patients accepted anti-fungal treatment and anti-retroviral treatment finally, the physician's mistakes reflect the rarity of this condition in China.
\end{abstract}

Conclusion: Cryptococcal prostatitis is a rare disease that unusually presents in immunodeficient patients. Physicians should have a heightened awareness of this particular infection in the immunodeficient population.

Keywords: Cryptococcal prostatitis, HIV, Tuberculosis

\section{Background}

Cryptococcosis is a common opportunistic infection in HIV-infected patients, causing up to $70 \%$ mortality at 3 months in some areas [1]. Patients with HIV tend to have more extraneural involvement and cryptococcemia than those without HIV [2]. Therefore, being aware of atypical Cryptococcus infection sites is critical when caring for HIV patients.

Cryptococcal prostatitis is rare. It usually presents in immunocompromised patients, such as those with diabetes, liver cirrhosis or organ transplantation [3-6]. Cryptococcus-infected prostates are occasionally reported in patients with AIDS [7, 8]. However, cryptococcal prostatitis has never been reported in China. Here, we report the first Chinese case of a patient with AIDS and urinary retention, in which the infection was misdiagnosed as tuberculosis before being correctly diagnosed as cryptococcal prostatitis.

\section{Case presentation}

In October 2017, a 47-year-old man had presented to a local hospital with cough and weight loss. He tested positive for HIV and a chest CT scan indicated a cavity in the right upper pulmonary lobe (Additional file 1). His $\mathrm{CD} 4^{+} \mathrm{T}$ cell count was 78 cells $/ \mu \mathrm{l}$ and his sputum

\footnotetext{
*Correspondence: xulijun1976@zju.edu.cn; xuli-jun@hotmail.com; zhubiao1207@zju.edu.cn

${ }^{1}$ National Clinical Research Center for Infectious Diseases, the First Affiliated Hospital, College of Medicine, Zhejiang University, Qingchun Rd, Hangzhou, China

Full list of author information is available at the end of the article
}

smear was negative for tuberculosis. He was diagnosed as HIV positive with tuberculosis infection (smear negative) and started antiviral therapy with tenofovir, lamivudine and efavirenz after 2 weeks of anti-tuberculosis treatment with isoniazid, rifampin, ethambutol and pyrazinamide.

In December 2017, the patient presented to the same hospital after having urinary retention for 2 weeks. He had no other symptoms such as headache, fever or cough. His prostate was found to be enlarged, and the doctor suggested continuing anti-tuberculosis treatment and inserted a urinary catheter to relieve the patient's urinary retention. Given that the urinary retention had deteriorated for two weeks before this visiting, the doctor suggested the patient attend the First Affiliated Hospital of Zhejiang University for better solution. After admission, physical examination revealed no significant clinical findings except for the presence of the urinary catheter. The sputum culture, smear and interferon- $\gamma$ release assay were all negative for $\mathrm{Myco-}$ bacterium tuberculosis. Lumbar puncture indicated a nearly normal cerebrospinal fluid (CSF) profile and the CSF was negative for cryptococcal antigen, so urinary retention as a result of central nervous system infection seemed unlikely. A CT scan indicated a cavity with effusion in the upper right lung (Fig. 1.a). Bronchoscopy was performed, and a lateral flow immunoassay and culture of the bronchoalveolar lavage fluid (BALF) were both positive for Cryptococcus neoformans. Enhanced MRI of the prostate revealed an abnormal patchy signal in the central gland of the prostate that was isointense 


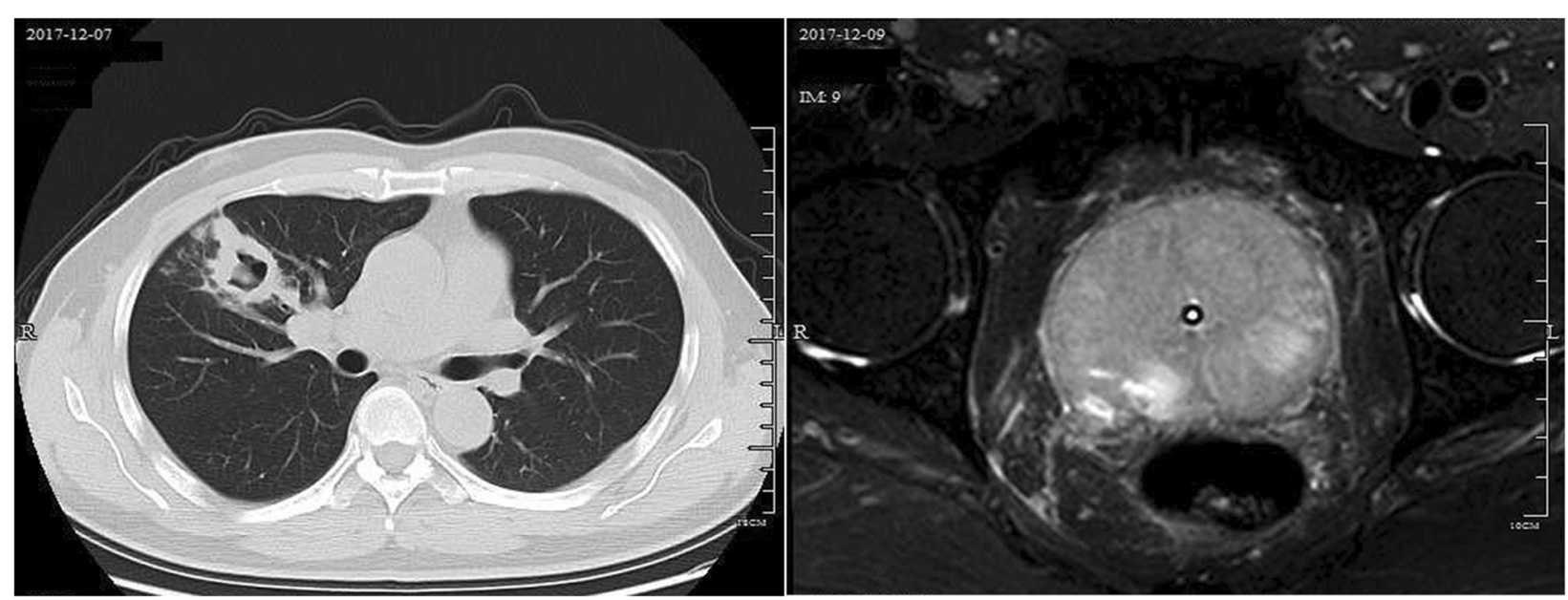

Fig. 1 Radiology images of patient. a. Chest CT in Dec 2017 indicated a cavity with effusion in the upper right lung; $\mathbf{b}$. Enhanced MRI of the prostate in Dec 2017 revealed an abnormal patchy signal in the central gland of the prostate

on T1-weighted imaging (WI), high intensity on T2WI, and no signal on diffusion-weighted imaging. The signal of T2 W1 was reduced in the seminal vesicles and peripheral zone of the prostate, and T1 W1 in the peripheral zone was enhanced (Fig. 1. b). An haematoxylin and eosin (H\&E) stain of the ultrasound-guided prostatic biopsy showed granulomatous inflammation; periodic acid-Schiff staining of the biopsied tissue was positive, Mayer's Mucicarmine stain was negative, Gomori-methenamine silver staining was positive, and Ziehl-Neelsen staining was negative (Fig. 2). Culture of the prostatic tissue grew Cryptococcus neoformans. The patient was diagnosed with AIDS with pulmonary cryptococcosis and cryptococcal prostatitis. Oral voriconazole (200 mg bid) combined with oral flucytosine $(100 \mathrm{mg} / \mathrm{kg} / \mathrm{d})$ for 2 months and then oral fluconazole $(600 \mathrm{mg} / \mathrm{d})$ for maintenance treatment for 4 months that relieved the cryptococcal prostatitis and urinary retention (Additional file 2).

\section{Discussion and conclusions}

China has a high prevalence of tuberculosis infection, and tuberculosis is a common opportunistic infection in Chinese HIV-infected patients. Smear-negative tuberculosis is more common in patients with HIV infection than in those without $[9,10]$. However, there have been reports of Cryptococcus infection in

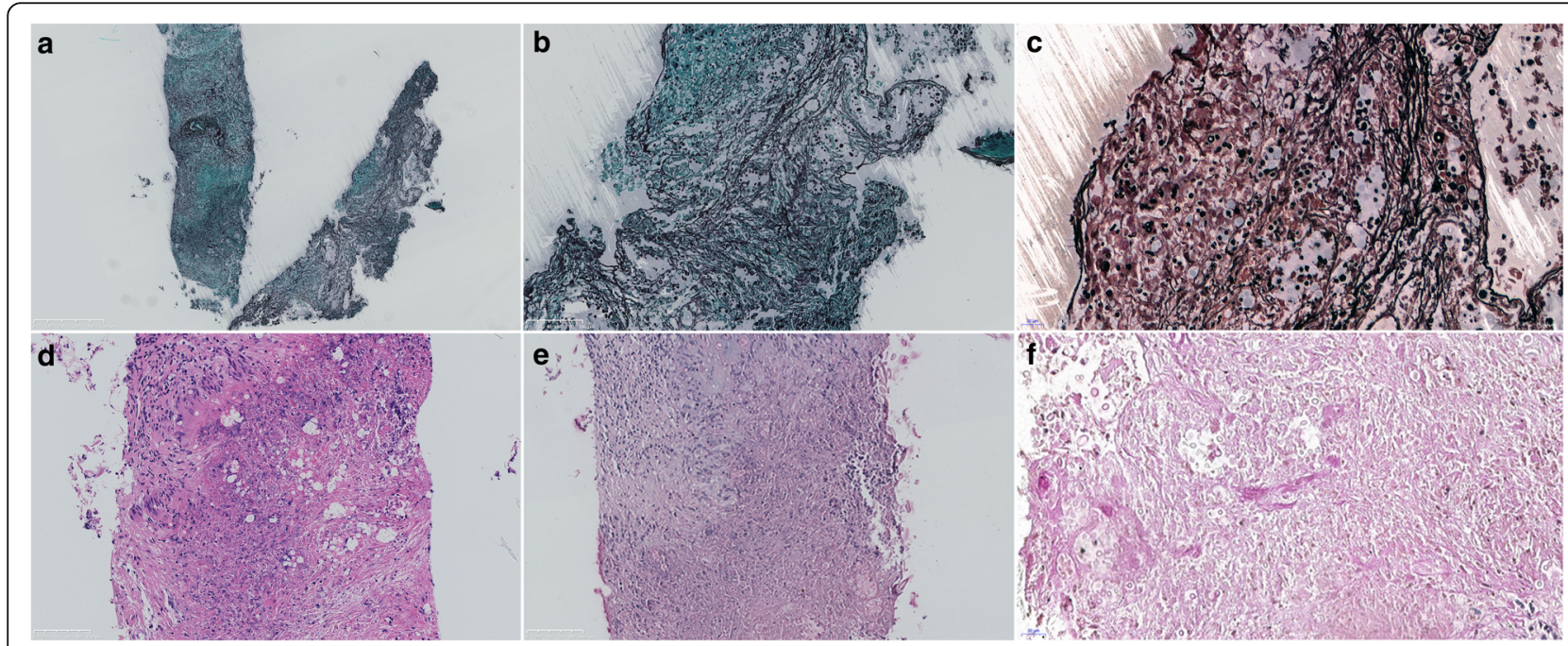

Fig. 2 Pathological finding in Prostate biopsy. Cryptococcal capsules were shown in histiocytes. a. Gomori-methenamine silver (GSM) staining (50x); b. GSM staining (200x); c. GSM staining (400x):d. Hematoxylin-Eosin (HE) staining (200x); d. Periodic Acid-Schiff (PAS) staining (200x); f. PAS staining $(400 x)$ 
immunocompromised patients with atypical presentations being misdiagnosed as tuberculosis [11, 12].

Our patient was misdiagnosed as having smearnegative pulmonary tuberculosis. Cryptococcus infection was hinted at by the lung cavity and the prostate hypertrophy; this was then confirmed by the presence of Cryptococcus in the BALF and prostatic biopsy specimen.

Although the correct diagnosis was reached eventually, this case provides some valuable lessons. First, a detailed pathogen screen should be done in HIV patients, especially in those with pneumonia; it is sometimes difficult to make accurate diagnoses based only on radiology. A lateral flow immunoassay for cryptococcal antigen is a rapid and reliable diagnosis method for suspected Cryptococcus infection [13]. Second, prostate infections should be a 'red flag' for urologists and pathologists in general hospitals in China. There are two types of hospitals in China responsible for HIV treatment; specialized and general hospitals. Doctors in some general hospitals, especially in those without HIV/AIDS wards, are less aware of atypical infections in HIV patients. Strengthening and highlighting AIDS-associated medical knowledge in these practitioners is important. Third, cryptococcal prostatitis is an uncommon disease in clinical practice, which may influence some doctors' correctly diagnosis and treatment decisions in some circumstances.

In summary, cryptococcal prostatitis is a rare disease that unusually presents in immunodeficient patients. Physicians should have a heightened awareness of this particular infection in the immunodeficient population.

\section{Supplementary information}

Supplementary information accompanies this paper at https://doi.org/10. 1186/s12879-019-4641-8.

Additional file 1. Chest $C T$ in Oct 2017

Additional file 2. Prostate $\mathrm{CT}$ after 6 month of anti-fungal treatment.

\section{Abbreviations}

AIDS: Acquired immune deficient syndrome; BLAF: Bronchoalveolar lavage fluid; CSF: Cerebrospinal fluid; CT: Computed tomography; HIV: Human immunodeficiency virus; MRI: Magnetic Resonance Imaging

\section{Acknowledgements}

Many thanks for works by the staffs in HIV/AIDS ward from the First Affiliated Hospital, College of Medicine, Zhejiang University.

\footnotetext{
Authors' contributions

LX drafted the manuscript and revised all versions; RT contributed to the care of the patient, QYZ contributed to prostatic biopsy, JLC provided pathological images and BZ revised the manuscript for publication. All authors read and approved the Manuscript.
}

\section{Funding}

1) The national science and technology major project of China during the 13th Five-year plan period (2018ZX10302104); 2) Independent Research Foundation of the State Key Laboratory for Diagnosis and Treatment of Infectious Diseases, the First Affiliated Hospital, College of Medicine, Zhejiang
University [491010*A61703]. The funding had no role in the study design, data collection and analysis, decision to publish, or preparation of the manuscript.

\section{Availability of data and materials}

Some original images were provided as supplements.

Ethics approval and consent to participate

Ethics approval or consent to participate was not applicable.

\section{Consent for publication}

Written consent to publish is obtained from study participant, and written consent is available for review by the Editor-in-Chief of journal.

\section{Competing interests}

The authors declare that they have no competing interests.

\section{Author details}

'National Clinical Research Center for Infectious Diseases, the First Affiliated Hospital, College of Medicine, Zhejiang University, Qingchun Rd, Hangzhou, China. ${ }^{2}$ The State Key Laboratory for Diagnosis and Treatment of Infectious Diseases, the First Affiliated Hospital, College of Medicine, Zhejiang University, No.79, Qingchun Rd, Hangzhou City 310003, China. ${ }^{3}$ Ultrasonography department, the First Affiliated Hospital, College of Medicine, Zhejiang University, Qingchun Rd, Hangzhou, China.

Received: 4 September 2019 Accepted: 19 November 2019 Published online: 12 December 2019

\section{References}

1. Limper AH, Adenis A, Le T, Harrison TS. Fungal infections in HIV/AIDS. Lancet Infect Dis. 2017;17(11):e334-e43. https://doi.org/10.1016/s14733099(17)30303-1.

2. Lee YC, Wang JT, Sun HY, Chen YC. Comparisons of clinical features and mortality of cryptococcal meningitis between patients with and without human immunodeficiency virus infection. J Microbiol Immunol Infect. 2011; 44(5):338-45. https://doi.org/10.1016/j.jmii.2010.08.011.

3. Brooks MH, Scheerer PP, Linman JW. CRYPTOCOCCAL PROSTATITIS. Jama. 1965;192:639-41.

4. Hinchey WW, Someren A. Cryptococcal prostatitis. Am J Clin Pathol. 1981; 75(2):257-60.

5. Siddiqui TJ, Zamani T, Parada JP. Primary cryptococcal prostatitis and correlation with serum prostate specific antigen in a renal transplant recipient. J Inf Secur. 2005;51(3):e153-7. https://doi.org/10.1016/j.jinf. 2004.12.005

6. Shah Sl, Bui H, Velasco N, Rungta S. Incidental finding of Cryptococcus on prostate biopsy for prostate adenocarcinoma following cardiac transplant: case report and review of the literature. Am J Case Rep. 2017:18:1171-80.

7. de Lima MA, dos Santos JA, Lazo J, Silva-Vergara ML, dos Santos LA, dos Santos VM. Cryptococcus infection limited to the prostate in an AIDS patient with disseminated mycobacteriosis. A necropsy report. Rev Soc Bras Med Trop. 1997:30(6):501-5.

8. Staib F, Seibold M, L'Age M, Heise W, Skorde J, Grosse G, et al. Cryptococcus neoformans in the seminal fluid of an AIDS patient. A contribution to the clinical course of cryptococcosis. Mycoses. 1989:32(4):171-80.

9. Floyd K, Glaziou P, Zumla A, Raviglione M. The global tuberculosis epidemic and progress in care, prevention, and research: an overview in year 3 of the end TB era. Lancet Respir Med. 2018;6(4):299-314. https://doi.org/10.1016/ s2213-2600(18)30057-2.

10. Maimaiti R, Zhang Y, Pan K, Mijiti P, Wubili M, Musa M, et al. High prevalence and low cure rate of tuberculosis among patients with HIV in Xinjiang, China. BMC Infect Dis. 2017;17(1):15. https://doi.org/10.1186/ s12879-016-2152-4.

11. Jarvis JN, Wainwright $H$, Harrison TS, Rebe K, Meintjes G. Pulmonary cryptococcosis misdiagnosed as smear-negative pulmonary tuberculosis with fatal consequences. Int J Infect Dis. 2010;14(Suppl 3):e310-2. https:// doi.org/10.1016/j.ijid.2010.02.2255

12. Ismail J, Chidambaram M, Sankar J, Agarwal S, Lodha R. Disseminated Cryptococcosis presenting as Miliary lung shadows in an Immunocompetent child. J Trop Pediatr. 2018;64(5):434-7. https://doi.org/ 10.1093/tropej/fm×083. 
13. Escandon P, Lizarazo J, Agudelo Cl, Chiller T, Castaneda E. Evaluation of a rapid lateral flow immunoassay for the detection of cryptococcal antigen for the early diagnosis of cryptococcosis in HIV patients in Colombia. Med Mycol. 2013;51(7):765-8. https://doi.org/10.3109/13693786.2013.781692.

\section{Publisher's Note}

Springer Nature remains neutral with regard to jurisdictional claims in published maps and institutional affiliations.

Ready to submit your research? Choose BMC and benefit from:

- fast, convenient online submission

- thorough peer review by experienced researchers in your field

- rapid publication on acceptance

- support for research data, including large and complex data types

- gold Open Access which fosters wider collaboration and increased citations

- maximum visibility for your research: over $100 \mathrm{M}$ website views per year

At BMC, research is always in progress.

Learn more biomedcentral.com/submissions 